\title{
Hydrodynamic impulse and energy of vortex tangle in superfluid turbulent He-II
}

\author{
S. K. Nemirovskii and V. A. Lushnikov \\ Institute of Thermophysics, Lavrentyeva Are. 1, 630090 Novosibirsk, Russia \\ E-mail: nemirov@otani.thermo.nsk.su
}

\begin{abstract}
The average hydrodynamic impulse (or Lamb impulse) $\mathbf{J}_{V}$ in the counterflowing, superfluid, turbulent He-II and the kinetic energy $E$, which is associated with the chaotic vortex filaments, are calculated. These quantities are defined as averages of the classical quantities taken over the stochastic vortex loop distribution. The averaging procedure was performed by using the Gaussian model of the chaotic vortex tangle elaborated earlier. Calculations show that the quantity $\mathbf{J}_{V}$ has an only a component in the direction of external counterflow velocity (created, e.g., by a heater). As for the energy, it is shown that besides the usual local contribution, the quantity $E$ includes additional terms due to a long-range interaction. Some dynamic properties of the turbulent He-II due to the impulse and energy of the vortex configuration, such as suppression of the superfluid density and decrease in the velocity of the second sound that propagates in the superfluid turbulent He-II, are discussed.
\end{abstract}

PASC: $67.40 .-w$

\section{Introduction}

It is widely appreciated that a chaotic tangle of quantized vortex filaments [vortex tangle (VT)] or superfluid turbulence (ST) appears in the superfluid component whenever the velocity of flow (or counterflow; see Fig. 1) exceeds a fairly small, critical value (see, e.g., Ref. 1). The presence of a vortex tangle essentially changes the hydrodynamic properties of superfluid helium (see, e.g., Refs. 2 and 3). Accordingly, the phenomena are studied in the framework of so-called phenomenological theory (PT) which was pioneered by Feynman [4] and Vinen [5] and greatly modified by Schwarz [6]. The PT describes ST in terms of the total length of vortex lines or vortex line density (VLD) $\mathcal{L}(t)$ and of the structure parameters of the VT. Knowledge of these quantities allows us to calculate some of the hydrodynamic characteristics of superfluid turbulent He-II such as mutual friction, sound attenuation, etc. There exist many other physical quantities connected with the distribution of the filaments and with their interaction, which is related to other physical phenomena that cannot be expressed in terms of the PT. The relevant phenomena should be covered by appropriate stochastic theory of chaotic vortex filaments. Of course, the most honest way to develop such a theory is to study stochastic dynamics of vortex filaments on the basis of the equations of motion with a source of chaos. However, due to extremely involved dynamics of vortex lines this method seems to be almost hopeless. We would like to offer another approach. The main idea and main strategy are the following. Despite the fact that

p

h

e $\mathrm{n}$ o $\mathrm{m}$ e $\mathrm{n}$ o

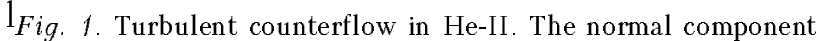
Clows from the heater carrying a heat flux $q=S T \mathbf{V}_{n}$; the sugerfluid component flows toward the heater. Total mass flow $\mathrm{j} \mathbf{j}=\rho_{n} \mathbf{V}_{n}+\rho_{s} \mathbf{V}_{n}=0$. Also shown are the axes used in this paper; the $z$ axis is directed along the normal velocity $\mathbf{V}_{n}$, the $x$ and $y$

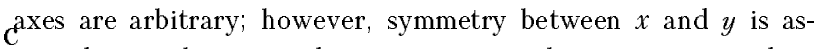
sumed. Usual measured quantities are the temperature drop or/and pressure, attenuation, and velocity of the second sound the at propagates at different angles through the counterflow, $t_{\text {shape of heat pulses, etc. }}$

h 


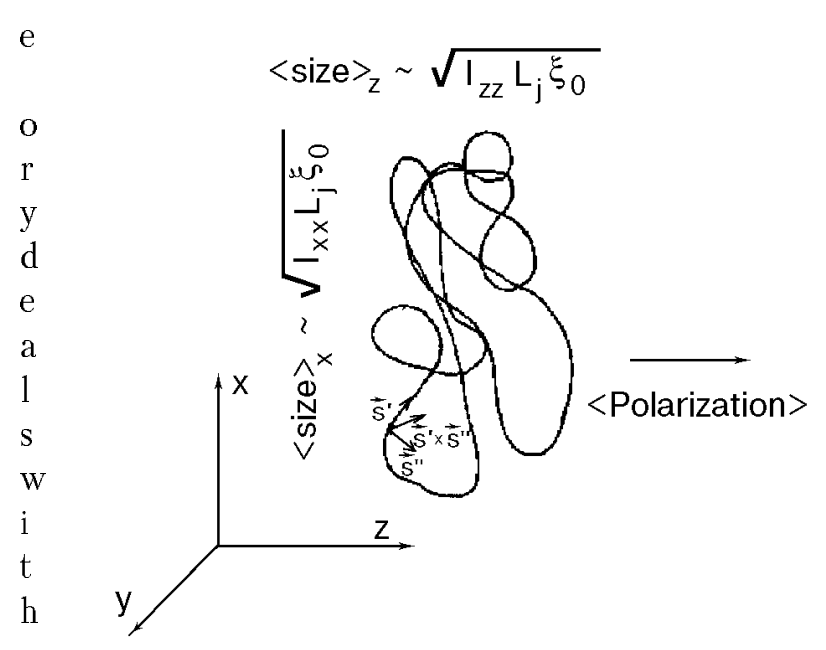

a

Fig. 2. A snapshot of the average vortex loop obtained from analysis of the statistical properties. The position of the vortex line element is described as $\mathbf{s}_{j}\left(\xi_{j}\right)$, where $\xi_{j}$ is the arc length, $\mathbf{s}_{j}^{\prime}\left(\xi_{j}\right)=d \mathbf{s}_{j}\left(\xi_{j}\right) / d \xi_{j}$ is the tangent vector, unit vector along the vortex line; $\mathbf{s}_{j}^{\prime \prime}\left(\xi_{j}\right)=d^{2} \mathbf{s}_{j}\left(\xi_{j}\right) / d \xi_{j}^{2}$ is the local curvature vector; vector production $\mathbf{s}_{j}^{\prime}\left(\xi_{j}\right) \times \mathbf{s}_{j}^{\prime \prime}\left(\xi_{j}\right)$ is binormal, which is responsible For the mutual orientation of the tangent vector and the vector \&f curvature. Close $(\Delta \xi<<R$, where $R$ is the mean curvature) eparts of the line are separated in $3 D$ space by a distance $\Delta \xi$.

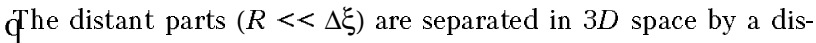
fance $(2 \pi R \Delta \xi)^{1 / 2}$ (with a correction due to the closeness). The latter property reflects a random walk structure of the vortex loops. As a whole, the loop is not isotropic and has a «pancake» shape. In addition, it has a total polarization $\left\langle\int \mathbf{s}_{j}^{\prime}\left(\xi_{j}\right) \times \mathbf{s}_{j}^{\prime \prime}\left(\xi_{j}\right) d \xi_{j}\right\rangle$, forcing the loop to drift along the vector $\mathbf{V}_{n}$ tand to produce nonzero superfluid mass flow in the $z$ direction $\mathrm{i}($ Sec. 3).

$\mathrm{t}$

y $\mathcal{L}(t)$, this approach, supplemented by experimental results and by some speculations, gives some information concerning the arrangement of the VT. We know that the VT consists of closed loops [labelled by $\mathbf{s}_{j}(\xi)$; see Fig. 2], which are uniformly distributed in space and which have the total length $\mathcal{L}(t)$ per unit volume. From acoustical experiments it follows that filaments are distributed in an anisotropic manner, and that quantitative characteristics of this anisotropy can be expressed by some structure parameters (see Refs. 1, 3, and 6). Besides this ordinary anisotropy, there is a more subtle anisotropy connected with the average polarization of the vortex loops. Furthermore, there is some proof that average curvature of the vortex lines is of the order of the inverse interline space and the coefficient of this proportionality was obtained in the numerical simulations made by Schwarz [6]. The master idea of my proposal is to construct a trial distribution function (TDF) in the space of the vortex loops of the most general form, which satisfies all of the established properties of the VT. We assume that this trial distribution function will enable us to calculate any physical quantities due to the VT.

In this paper we calculate the average hydrodynamic impulse (or Lamb impulse) $\mathbf{J}_{V}$ in the counterflowing, superfluid, turbulent He-II and the average kinetic energy $E$ which is associated with the chaotic vortex filaments.

\section{Construction of the trial distribution function}

According to general prescriptions, the average of any quantity $\left\langle\mathcal{B}\left(\left\{\mathbf{s}_{j}\left(\xi_{j}\right)\right\}\right)\right\rangle$, which depends on the vortex loop configurations, is

$$
\left\langle\mathcal{B}\left(\left\{\mathbf{s}_{j}\left(\xi_{j}\right)\right\}\right)\right\rangle=\sum_{\left\{\mathbf{s}_{j}\left(\xi_{j}\right)\right\}} \mathcal{B}\left(\left\{\mathbf{s}_{j}\left(\xi_{j}\right)\right\}\right) \mathscr{P}\left(\left\{\mathbf{s}_{j}\left(\xi_{j}\right)\right\}\right) .
$$

Here $\mathcal{P}\left(\left\{\mathbf{s}_{j}\left(\xi_{j}\right)\right\}\right)$ is the probability of the vortex tangle to have a particular configuration $\left\{\mathbf{s}_{j}\left(\xi_{j}\right)\right\}$. The meaning of summation over all vortex loop configurations $\sum_{\left\{\mathbf{s}_{j}\left(\xi_{j}\right)\right\}}$ in Eq. (1) will be clear from further analysis.

We make the usual assumption in statistical physics that all configurations corresponding to the same macroscopic state have equal probabilities. Thus, the probability $\mathscr{P}\left(\left\{\mathbf{s}_{j}\left(\xi_{j}\right)\right\}\right)$ for vortex tangle to have a particular configuration $\left\{\mathbf{s}_{j}\left(\xi_{j}\right)\right\}$ should be proportional to $1 / N_{\text {allowed }}$, where $N_{\text {allowed }}$ is the number of allowed configurations, which, of course, is infinite:

$$
\mathcal{P}\left(\left\{\mathbf{s}_{j}\left(\xi_{j}\right)\right\}\right) \propto \frac{1}{N_{\text {allowed }}} .
$$

By «allowed configurations» $N_{\text {allowed }}$ we mean only the configurations that lead to the correct values for all average quantities known from experiment and numerical simulations.

Formally, it can be expressed as a path integral in the space of three-dimensional (closed) curves supplemented with some constraints connected to the properties of the VT:

$N_{\text {allowed }} \propto \prod_{j} \mathcal{D}\left(\left\{\mathbf{s}_{j}(\xi)\right\}\right) \cdot$ constraints $\left\{\mathbf{s}_{j}(\xi)\right\}$

The constraints entering this relation are expressed by delta functions that give fixed properties of the VT. For instance, the constraint $\delta\left(\left(\mathbf{s}_{j}^{\prime}(\xi)^{2}-1\right)\right.$ shows that the parameter $\xi$ is the arc length. However, this condition leads to an intractable theory. We will use a trick known from the theory of polymer chains (see, e.g., Ref. 7); namely, we will relax the rigorous condition and change the delta function by continuous (Gaussian) distribution of 
the link length with the same value of integral. This trick leads to the expression for the number of allowed configurations

$$
N_{\text {allowed }} \propto \prod_{j} \mathcal{D}\left(\left\{\mathbf{s}_{j}(\xi)\right\}\right) \exp \left(-\lambda_{1} \int_{0}^{\mathcal{L}}\left|\mathbf{s}^{\prime}\right|^{2} d \xi\right) .
$$

In the same manner we are able to introduce other known properties of the VT structure. The detailed calculations will be published elsewhere [8]. We can now write the final expression for the probability of configurations

$$
N_{\text {allowed }} \propto \int \mathcal{D}\{\mathbf{s}(\kappa)\} \exp (-\mathcal{L}\{\mathbf{s}(\kappa)\}) .
$$

Here $\mathbf{s}(\kappa)$ is a one-dimensional Fourier transform ${ }^{*}$ of the variable $\mathbf{s}(\xi)$, and the Lagrangian $\mathcal{L}\{\mathbf{s}(\kappa)\}$ is

$$
\mathcal{L}\{\mathbf{s}(\kappa)\}=\sum_{\kappa \neq 0} \mathbf{s}_{x}^{\alpha}(\kappa) \Lambda^{\alpha \beta}(\kappa) \mathbf{s}_{x}^{\beta}(\kappa) .
$$

In practice, to calculate various averages it is convenient to work with the characteristic (generating) functional (CF), which is defined as the average

$$
W\left(\left\{\mathbf{P}_{j}(\kappa)\right\}\right)=\left\langle\exp \left(-\sum_{j} \sum_{\kappa \neq 0} \mathbf{P}_{j}^{\mu}(\kappa) \mathbf{s}_{j}^{\prime \mu}(-\kappa)\right)\right\rangle .
$$

Accordingly, our Lagrangian has a quadratic form [in $\mathbf{s}(\kappa)$ ]. Consequently, the trial distribution function is a Gaussian function, calculation of the CF can be made by using a full square procedure to give the result

$$
W\left(\left\{\mathbf{P}_{j}(\kappa)\right\}\right)=\exp \left(-\sum_{j} \sum_{\kappa \neq 0} \mathbf{P}_{j}^{\mu}(\kappa) N_{j}^{\mu \nu}(\kappa) \mathbf{P}_{j}^{v}(-\kappa)\right) .
$$

The elements of the matrix $N_{j}^{\mu \nu}(\kappa)$ are specified from calculation of the total length, anisotropy coefficient, curvature, and polarization. Thier explicit form is written in Ref. 8. The typical shape of the averaged curve is shown in Fig. 2.

Thus we reached our goal and have written the expression for the trial $\mathrm{CF}$, which enables us to calculate any average vortex filament configuration.

\section{Hydrodynamic impulse of the vortex tangle}

As an illustration of the developed theory we would like to discuss hydrodynamic impulse of $\mathrm{V}$

o

$\mathrm{r}_{*}$ We used $1 D$ Fourier transform $\mathbf{s}_{j}(\kappa)$ of curves $\mathbf{s}_{j}(\xi)$ since various properties of the VT, such as the mean curvature, polarization, anisotropy, etc. are expressed via the derivatives of $\mathbf{s}_{j}(\xi)$ of different orders. tex tangle $\mathbf{J}_{\mathbf{V}}$ which is defined as

$$
\mathbf{J}_{\mathbf{V}}=\left\langle\frac{\rho_{s} \tilde{\kappa}}{2} \sum_{j} \int \mathbf{s}_{j}\left(\xi_{j}\right) \times \mathbf{s}_{j}^{\prime}\left(\xi_{j}\right) d \xi_{j}\right\rangle .
$$

The quantity $\mathbf{J}_{\mathbf{V}}$ is closely related to the momentum of the fluid (see Ref. 9). The averaged $\left\langle\mathbf{s}_{j}\left(\xi_{j}\right) \times \mathbf{s}_{j}^{\prime}\left(\xi_{j}\right)\right\rangle$ is immediately evaluated by CF (7) to give the result

$$
\mathbf{J}_{\mathbf{V}}^{z}=-\left[\frac{\rho \tilde{\kappa} I_{l} \alpha_{v}}{\rho_{n} c_{2}^{2} \beta_{v}}\right] \rho_{s} \mathbf{V}_{s}
$$

Note that the coefficient includes no fitting parameters but only the characteristics known from the phenomenological theory (see Ref. 6). The relation (9) shows that the vortex tangle induces the superfluid current directed against the external superfluid current. This should be expected since there is a preferable polarization of the vortex loops. In the experiments this additional superfluid current manifests itself as a suppression of the superfluid density. This effect is a $3 D$ analog of the well-known Kosterlitz-Thouless effect except that distribution of the vortex lines is not calculated but is written with reference to the experimental data.

Since superfluid density enters the expression for second-sound velocity, it seems attractive to detect it using transverse second sound testing (Fig. 3). To do this, we must first evaluate transverse change of the $\rho_{s}$ and, secondly, develop the theory to match it to the nonstationary case. The general theory asserts that while applying a harmonic external second-sound field, suppression of superfluid density $\mathrm{b}$

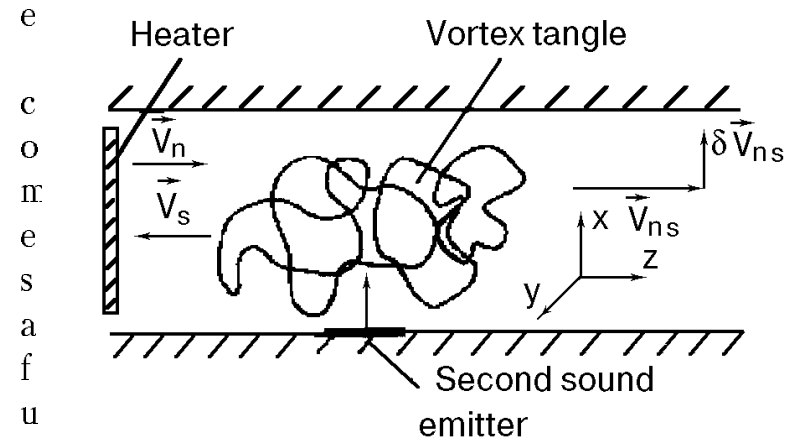

$\mathrm{n}$

Fig. 3. Illustration of the transverse experiment. Small perpendicular deviation $\delta \mathbf{V}_{n s}$ of the counterflow velocity $\mathbf{V}_{n s}$ changes the orientation of polarization of the vortex tangle, whereas the $t$ vortex line density and mean curvature do not change in the first order in $\delta \mathbf{V}_{n s}$. 
ion of frequency $\omega$ in the form

$$
\Delta \rho_{s}^{x}(\omega)=\left(\frac{\delta \mathbf{J}_{V}^{x}}{\delta \mathbf{V}_{s}^{x}}\right)_{\text {transv }} \frac{1}{1+i \omega \tau_{J}} .
$$

Here the transverse $\left(\delta \mathbf{J}_{V}^{x} / \delta \mathbf{V}_{s}^{x}\right)_{\text {transv }}$ is half that given by relation (9). The quantity $\tau_{J}$ is the time of relaxation of the superfluid current $J_{V}$, which can be found from dynamical considerations. Expressing $d \mathbf{J}_{V}^{x} / d t$ with the help of the equation of motion of the vortex line elements and then evaluating various averages by our trial distribution function, we obtain the following final result for the change of the second-sound velocity.

Performing all of the described procedures, we find that the relative change is

$$
\Delta u_{2} / u_{2}=-f(T)\left(\mathbf{V}_{n s}^{4} / \omega^{2}\right) .
$$

Here the function $f(T)$ consists of the structure parameters of the vortex tangle

$$
f(T)=\frac{4 \rho \tilde{\kappa} I_{l}^{2} \alpha^{2}\left(1-I_{x x}\right)^{2}}{\rho_{n} c_{2}^{4} \beta^{3}} .
$$

Decreasing the second-sound velocity in the counterflowing He-II has been actually observed about two decades ago by Vidal et al. [10]. To the best of our knowledge, there was only an attempt to consider this effect theoretically by Mehl [11], who explained the change in the second-sound velocity by introducing the imaginary part into the HallVinen constant. However, aside from some numerical disagreement, the Mehl's theory did not explain the strong $\mathbf{V}_{n s}$ dependence in the experimental data. Let us compare our result (11) with Vidal's experiment. Using the data on the structure parameters, we find that, e.g., at the temperature $1.44 \mathrm{~K}$ the value of the function $f(T)$ is about $620 \mathrm{~s}^{2} / \mathrm{cm}^{4}$. Using the frequency $\omega=4.3 \mathrm{rad} / \mathrm{s}$ in Ref. 10 and $V_{n s}=2 \mathrm{~cm} / \mathrm{s}$, we find that $\Delta u_{2} / u_{2} \approx 4 \cdot 10^{-4}$, which is very close to the observed value.

\section{Energy of the vortex tangle}

In this section we calculate the average energy of the stochastic vortex loop distributed according to the distribution function (5). The general expression associated with the linear vortices can be written as follows (see, e.g., Ref. 9):

$$
E=\left\langle\frac{1}{2} \int \rho_{s} \mathbf{v}_{s}^{2} d^{3} \mathbf{r}\right\rangle=
$$

$$
=\left\langle\frac{\rho_{s} \kappa^{2}}{8 \pi} \sum_{j, i} \int_{0}^{L_{i}} \int_{0}^{L_{j}} \frac{\mathbf{s}_{i}^{\prime}\left(\xi_{i}\right) \mathbf{s}_{j}^{\prime}\left(\xi_{j}\right)}{\left|\mathbf{s}_{i}\left(\xi_{i}\right)-\mathbf{s}_{j}\left(\xi_{j}\right)\right|} d \xi_{i} d \xi_{j}\right\rangle .
$$

In $3 D$ Fourier space the average energy $E$ (13) can be rewritten as

$$
\begin{gathered}
E=\left\langle\frac{\rho_{s} \kappa^{2}}{2} \sum_{i, j} \int_{\mathbf{k}} \frac{d^{3} \mathbf{k}}{(2 \pi)^{3} \mathbf{k}^{2}} \int_{0}^{L_{i} \int_{j}} \mathbf{s}_{j}^{\prime}\left(\xi_{i}\right) \mathbf{s}_{j}^{\prime}\left(\xi_{j}\right) d \xi_{i} d \xi_{j} \times\right. \\
\quad \times \exp \left(i \mathbf{k}\left(\mathbf{s}_{i}\left(\xi_{i}\right)-\mathbf{s}_{j}\left(\xi_{j}\right)\right)\right\rangle .
\end{gathered}
$$

Comparing (14) and (7), it is possible to express the energy $E$ in terms of the characteristic functional:

$$
\langle E\rangle=\frac{\rho_{s} \kappa^{2}}{2} \sum_{i, j} \int_{\mathbf{k}} \frac{d^{3} \mathbf{k}}{(2 \pi)^{3} \mathbf{k}^{2}} \times
$$

$$
\times \int_{0}^{L_{i} \int_{j}} d \xi_{i} d \xi_{j} \frac{\delta^{2} W}{i \delta \mathbf{P}_{i}^{\alpha}\left(\xi_{i}\right) i \delta \mathbf{P}_{j}^{\alpha}\left(\xi_{j}\right)} \exp \left[i \mathbf{k}\left(s_{i}(0)-s_{j}(0)\right)\right] .
$$

Here the set of $\mathbf{P}_{n}\left(\xi_{n}^{\prime}\right)$ in CF $W\left(\left\{\mathbf{P}_{n}\left(\xi_{n}^{\prime}\right)\right\}\right)$ is again determined with the help of the $\theta$-functions

$$
\begin{gathered}
\mathbf{P}_{i}\left(\xi_{i}^{\prime}\right)=\mathbf{k} \theta\left(\xi_{i}^{\prime}\right) \theta\left(\xi_{i}-\xi_{i}^{\prime}\right), \mathbf{P}_{j}\left(\xi_{j}^{\prime}\right)=\mathbf{k} \theta\left(\xi_{j}^{\prime}\right) \theta\left(\xi_{j}-\xi_{j}^{\prime}\right), \\
\mathbf{P}_{n}\left(\xi_{n}\right)=0, n \neq i, j .
\end{gathered}
$$

The relation (16) implies that we choose in the integrand in the exponent of $\mathrm{CF}$ only the points lying in the interval from 0 to $\xi_{i}$ on the $i$-curve and from 0 to $\xi_{j}$ on the $j$-curve. In the evaluation of self-energy of the same loop, $i=j$, one has to distinguish the points $\xi_{i}$, and assume them to be, e.g., $\xi_{i}^{\prime}$ and $\xi_{i}^{\prime \prime}$. Further results concern the case of the only loop of length $L$. Omitting cumbersome calculations, we write the final result in the form

$$
\begin{gathered}
E=\frac{\rho \kappa^{2} L}{4 \pi} \ln \frac{R}{a_{0}}+\frac{\rho \kappa^{2} L}{4 \pi}\left(1-\frac{2}{\sqrt{4}}\left(f_{2}-f_{1}\right)\right) \ln \frac{R}{a_{0}}+ \\
+\frac{\rho \kappa^{2} L}{4 \pi}\left[\frac{2 f_{3}}{(\sqrt{\pi}-1)^{1 / 2} \pi^{5 / 2} c_{23}^{2}} I_{l}^{2}+\frac{f_{2}}{\pi^{3 / 2}(\sqrt{\pi}-1)^{1 / 2}}\right],
\end{gathered}
$$

where the quantities $f$ are expressed in terms of the structure parameters of the VT as follows: 


$$
\begin{gathered}
f_{1}(\beta)=\left[2\left(3-\beta^{2}\right)\right]^{1 / 2} \frac{\arcsin \beta}{\beta}, \\
f_{2}(\beta)=\frac{1}{\left[2\left(3-\beta^{2}\right)\right]^{1 / 2}}\left(-\sqrt{1-\beta^{2}}+\left(2-\beta^{2}\right) \frac{\arcsin \beta}{\beta}\right) \\
f_{3}(\beta)=\left[2\left(3-\beta^{2}\right)\right]^{3 / 2}\left(\frac{\sqrt{1-\beta^{2}}}{\beta^{2}}-\frac{\arcsin \beta}{\beta^{3}}\right) \\
\beta=\sqrt{\left(I_{x}-I_{z}\right) / I_{x}} .
\end{gathered}
$$

Let us examine expression (17). The first term on its right side is the energy per unit length of the straight vortex filament (see, e.g., Ref. 1) multiplied by its length. In this form it is frequently used in the theory of superfluid turbulence (see, e.g., Ref. 3). But there are additional terms. The second term is also logarithmically large. We assume that this contribution somes from accidental self-crossing of remote (along the line) parts of the vortex filament. The third and forth terms result from long-range interaction; they are smaller (about 10\%) than the logarithmic terms. The third term is of special interest. It appears due to the polarization of the vortex loop and its presence implies that there is some elasticity of the vortex tangle in the $\mathbf{V}_{n s}$ direction. The results of the previous section show that the presence of the VT leads to the appearance of a correction of the superfluid density. Therefore, combination of the longitudinal elasticity and inertia of the «joint» mass should lead to the appearance of elastic waves, $3 D$ analog of the Tkachenko waves.

\section{Conclusion}

Based on the trial distribution function in the space of vortex loops comprising a superfluid turbulence in He-II a hydrodynamic impulse, as well as energy of vortex tangle. Some dynamic properties of the turbulent He-II, due to the impulse and energy of vortex configuration such as suppression of the superfluid density and decrease in the velocity of the second sound that, propagate in the superfluid turbulent He-II, have been discussed.

This work was funded in part by the Russian Foundation for Basic Research, Grant N 96-02-19414.

1. R. J. Donnelly, Quantized Vortices in Helium II, Cambridge University Press (1991).

2. J. T. Tough, in: Progress in Low Temperature Physics, D. F. Brewer (ed.), North Holland (1982), p. 133.

3. S. K. Nemirovskii and W. Fiszdon, Rev. Mod. Phys. 67, No.1, 37 (1995).

4. R. P. Feynman, in: Progress in Low Temperature Physics, C. J. Gorter ( ed.), North Holland (1955), vol. 1, p. 17.

5. W. F. Vinen, Proc. R. Soc. London A240, 114 (1957); ibid. A240, 128 (1957); ibid. A242, 493 (1957); ibid. A243, 400 (1958).

6. K. W. Schwarz, Phys. Rev. B18, 245 (1978); ibid. B31, 5782 (1985); ibid. B38, 2398 (1988).

7. M. Doi and S. F. Edwards, The Theory of Polymer Dynamics, Clarendon Press, Oxford (1986).

8. S. K. Nemirovskii, Chech. J. Phys., Vol 46 (Suppl.S1) (1996), Phys. Rev. B (submitted).

9. G. K. Batchelor, An Introduction to Fluid Mechanics, Cambridge University Press (1967).

10. F. C. Vidal, C. R. Acad Sci. B275, 609 (1972).

11. J. B. Mehl, Phys. Rev. A10, 601 (1974). 